\title{
Multiple Coils for Reduction of Flow Artefacts in MR Images
}

\author{
David Atkinson ${ }^{1}$, David J. Larkman ${ }^{2}$, Philipp G. Batchelor ${ }^{1}$, Derek L.G. Hill ${ }^{1}$, \\ and Joseph V. Hajnal ${ }^{2}$ \\ 1 Imaging Sciences, Guy's Hospital, King's College London, SE1 9RT, UK, \\ David.Atkinson@kcl.ac.uk, \\ 2 Robert Steiner MRI Unit, Imaging Sciences Department, Clinical Sciences Centre, \\ Hammersmith Hospital, Imperial College London, W12 0NN, UK.
}

\begin{abstract}
Flowing blood can cause streak or blob artefacts in MR images and these may degrade subsequent image analysis. Multiple MRI receiver coils enable the reconstruction of images using data from different combinations of coils. The artefact intensities differ in these images due to the differing coil sensitivities. The artefact cause is parameterised and an optimisation routine is used to find self-consistent image reconstructions which have reduced artefacts.
\end{abstract}

\section{Introduction}

Blood flow and other physiological processes can result in the ghosting and blurring of MR images. In addition to the blurring, these artefacts can introduce additional features that are not present in the object. This can be problematic, for example, difference images from registered serial brain volumes can be corrupted by flow artefacts from the carotid and middle cerebral arteries.

Multiple coils have previously been used to detect and reject motioncorrupted phase encode lines [1. The remaining data was then reconstructed using a generalised SMASH [2] approach. Odd and even echos in EPI images have been used for flow artefact reduction [3. Here we apply a new, more general, technique to the problem of artefacts from flowing blood.

\section{Method}

In the absence of any artefacts, the image $s^{\gamma}$ obtained from coil $\gamma$ with spatial sensitivity $c^{\gamma}$ is given by

$$
s^{\gamma}=c^{\gamma} \cdot r
$$

where $r$ is the underlying object. This can be expressed in k-space as a convolution which in turn can be written as the matrix multiplication $\mathbf{S}=\mathbf{C R}$. Here $\mathbf{S}$ and $\mathbf{C}$ can contain data relating to either one or all coils. In the presence of flow artefacts, the k-space representation of the object and the acquired k-space change to $\overline{\mathbf{R}}$ and $\overline{\mathbf{S}}$ respectively. If the artefact can be parameterised as a change 
to the coils, rather than the object, we can write $\overline{\mathbf{S}}=\mathbf{C} \overline{\mathbf{R}}=\overline{\mathbf{C}} \mathbf{R}$. In the case of flowing blood, we assume that the coil profile at the position of the artery can be multiplied by one complex term for each discrete time step during the scan. Solving for $\mathbf{R}$ with one or all coils [2] enables us to minimise the cost function

$$
F=\sum_{\gamma, n}\left|r^{\gamma}-r^{G}\right|^{2},
$$

where the reconstructed object has been Fourier transformed to the image domain and the sum is over all coils and all image-domain pixels.

\section{Results and Conclusions}

Figure 1 shows an axial slice from a volunteer acquired on a Philips 1.5T Eclipse using a 4-element body array coil $(128 \times 128$, TR/TE of 250/10 ms, breath-hold, no cardiac gating, 128 discrete time points). Ghosts can be seen in the spinal body and intestine region as well as outside the body. We use the MATLAB [The MathWorks] least squares nonlinear optimisation routine with 256 unknowns (128 complex multiplicative factors). After processing with our algorithm, the ghosts are reduced and image quality is comparable to a similar slice acquired with saturation bands (which would not be effective for multi-slice acquisitions).
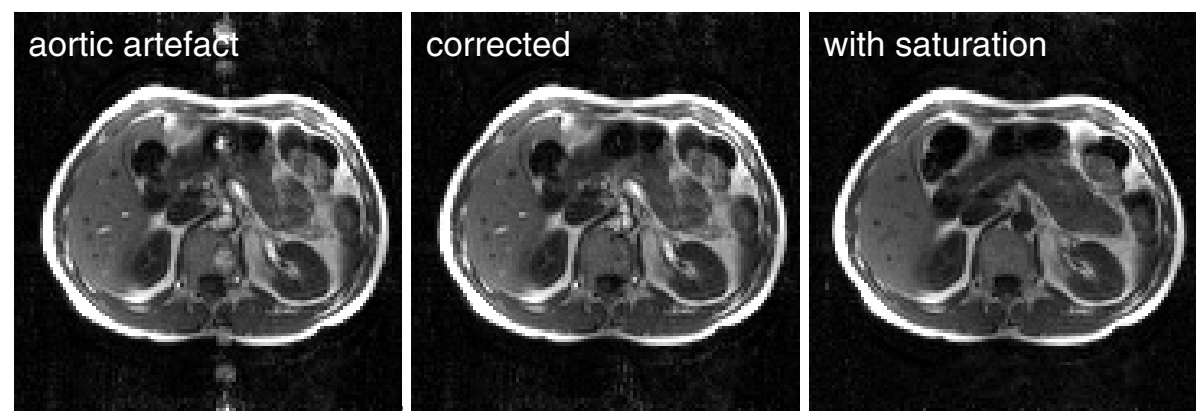

Fig. 1. Slice corrupted by flow in aorta (left), corrected (middle) and a similar slice acquired using saturation slabs in a different breath-hold (right).

\section{References}

1. M. Bydder et al. Magn. Reson. Med., 47:677-686, 2002.

2. M. Bydder et al. Magn. Reson. Med., 47:160-170, 2002.

3. Y. Mashida et al. Proc. ISMRM 9(2001), p. 1810. 